Revue musicale OICRM

\title{
Enjeux politiques et espaces médiatiques dans les écrits de compositeurs polonais
}

\section{Stefan Keym}

Volume 7, numéro 1, 2020

URI : https://id.erudit.org/iderudit/1069478ar

DOI : https://doi.org/10.7202/1069478ar

Aller au sommaire du numéro

\section{Éditeur(s)}

Observatoire interdisciplinaire de création et recherche en musique (OICRM)

\section{ISSN}

2368-7061 (numérique)

Découvrir la revue

Citer cet article

Keym, S. (2020). Enjeux politiques et espaces médiatiques dans les écrits de compositeurs polonais. Revue musicale OICRM, 7(1), 168-178.

https://doi.org/10.7202/1069478ar
Résumé de l'article

Les stratégies créatrices et médiatiques des compositeurs polonais furent fortement influencées par la situation politique particulière de leur pays, longtemps occupé par des puissances étrangères. L'engagement patriotique que la société polonaise exigeait de ses artistes permit à quelques compositeurs et à leurs textes de pénétrer dans des espaces médiatiques au-delà du domaine artistique. Les enjeux entre patriotisme et autopromotion artistique sont analysés à travers les écrits de compositeurs polonais de l'époque avant et après la renaissance de l'état polonais en 1918. Alors qu'avant cette césure la musique et le discours autour d'elle servaient souvent de moyen à des fins politiques (notamment dans le cas d'Ignacy Jan Paderewski), on constate une situation opposée aux années 1920, lorsque Karol Szymanowski n’hésita pas à instrumentaliser le sentiment nationaliste afin de promouvoir sa propre nouvelle esthétique comme une sorte de " libération » du joug de la musique germanique du XIX ${ }^{\mathrm{e}}$ siècle. 


\title{
Enjeux politiques et espaces médiatiques dans les écrits de compositeurs polonais \\ Stefan Keym
}

\begin{abstract}
Résumé
Les stratégies créatrices et médiatiques des compositeurs polonais furent fortement influencées par la situation politique particulière de leur pays, longtemps occupé par des puissances étrangères. L'engagement patriotique que la société polonaise exigeait de ses artistes permit à quelques compositeurs et à leurs textes de pénétrer dans des espaces médiatiques au-delà du domaine artistique. Les enjeux entre patriotisme et autopromotion artistique sont analysés à travers les écrits de compositeurs polonais de l'époque avant et après la renaissance de l'état polonais en 1918. Alors qu'avant cette césure la musique et le discours autour d'elle servaient souvent de moyen à des fins politiques (notamment dans le cas d'Ignacy Jan Paderewski), on constate une situation opposée aux années 1920, lorsque Karol Szymanowski n'hésita pas à instrumentaliser le sentiment nationaliste afin de promouvoir sa propre nouvelle esthétique comme une sorte de "libération " du joug de la musique germanique du XIX ${ }^{\mathrm{e}}$ siècle.
\end{abstract}

Mots clés : écrits de compositeurs ; musique et nationalisme ; musique polonaise ; querelles sur la musique; revues musicales.

\begin{abstract}
The creative and media strategies of Polish composers were strongly influenced by the political situation of their country, occupied by foreign powers for a long time. The patriotic engagement which Polish society demanded from its artists, enabled some composers and their texts to reach media spaces beyond the field of art. The interactions between patriotism and artistic self-promotion are analyzed at the example of the writings of Polish composers from the time before and after the renaissance of the Polish state in 1918. Whereas before this caesura music and the discourse about it often served as means for political purposes (especially in the case of Ignacy Jan Paderewski), the opposite is to be observed in the 1920s when Karol Szymanowski did not hesitate to instrumentalize nationalist feelings in order to promote his own new aesthetics as a sort of "liberation" from the yoke of $19^{\text {th }}$-century Germanic music.
\end{abstract}

Keywords: debates on music; music and nationalism; music periodicals; Polish music; writings of composers. 
$\mathrm{Au} \mathrm{XIX}^{\mathrm{e}}$ siècle, les stratégies créatrices et médiatiques de compositeurs polonais furent fortement influencées par la situation politique très particulière de leur pays, occupé et divisé par trois puissances étrangères (la Russie, la Prusse et l'Autriche). L'élite de la société polonaise exigeait de ses artistes un engagement patriotique, sur le modèle d'écrivains tels qu'Adam Mickiewicz, afin de préserver l'identité nationale. Cet horizon d'attente concernait non seulement les œuvres musicales, mais encore les écrits et les propos énoncés publiquement par les musiciens.

Or, la mission patriotique des musiciens polonais appelait une grande souplesse des auteurs face à la censure, notamment dans les zones russe et prussienne. Néanmoins, cette mission permit à quelques compositeurs de pénétrer avec leurs textes dans des espaces médiatiques larges, au-delà du domaine purement artistique. La politisation du discours musical polonais avait donc des effets ambigus : d'un côté, des musiciens instrumentalisaient leur art à des fins politiques ; de l'autre, des arguments politiques pouvaient aussi servir de moyen pour imposer une nouvelle esthétique.

L'étude du cas de la Pologne avant et après sa résurrection politique en novembre 1918 nous permettra de discuter quelques problèmes généraux liés aux aspects politiques dans des écrits de compositeurs, ainsi qu'à leur diffusion médiatique.

Évoquons d'abord brièvement l'état de la question. S'il existe des éditions critiques des écrits de quelques compositeurs polonais renommés (Szymanowski 1984 et 1989 ; Lutosławski 2011), des anthologies de prises de position de musiciens polonais sur des sujets divers (Jarociński 1955, Tomaszewski 1959, Demska-Trębacz 1991) et une monographie brillante sur la critique musicale polonaise (Dziadek 2002a et b), il manque des études focalisées sur le problème des écrits de compositeurs. D'ailleurs, le plus célèbre de ces derniers, Frédéric Chopin, refusait de s'exprimer à travers des écrits et il en était de même pour Stanisław Moniuszko (1819-1872), l'auteur de l'opéra national polonais Halka (1858).

\section{NOSKOWSKI : AU SERVICE DE LA MUSIQUE INSTRUMENTALE}

Le premier compositeur polonais à avoir publié un corpus considérable de textes fut Zygmunt Noskowski (1846-1909), disciple de Moniuszko et compositeur le plus influent à Varsovie à partir de 1880. Comme beaucoup de ses collègues polonais, il avait fait des études à Berlin, auprès de Friedrich Kiel (Keym 2010). C'est là qu'il connut une sorte de conversion à la musique instrumentale dite sérieuse. Or, il n'y avait pas d'orchestre symphonique permanent en Pologne avant 1901. Afin d'y implanter la musique instrumentale, Noskowski s'engagea simultanément en tant que compositeur, chef d'orchestre, journaliste et pédagogue. Il occupa les postes de professeur de composition au Conservatoire et de directeur de la Société musicale de Varsovie. La plupart des compositeurs de la génération suivante devinrent ses disciples.

Hormis un traité de contrepoint (Noskowski 1907) et quelques paratextes pour ses œuvres symphoniques à programme, le corpus des écrits de Noskowski consiste surtout en des critiques musicales et d'autres articles qu'il publia entre 1870 et 1909 dans divers périodiques : des quotidiens et des revues musicales, culturelles et générales. L'objectif principal de ces articles était d'initier les lecteurs polonais aux principes esthétiques de la "musique sérieuse ». Par exemple, dans une série d'articles intitulée 
"De Bach à Chopin ", Noskowski présenta son canon des grands compositeurs qu'il compara avec une chaîne de montagnes majestueuses (Noskowski 1892). Ici et ailleurs, il mettait l'accent sur la musique instrumentale. Même sa série d'articles sur «L'opéra idéal » commence par l'hypothèse selon laquelle le genre musical le plus noble serait la symphonie - une affirmation provocatrice, car peu répandue en Pologne à l'époque (Noskowski 1888b).

Or, l'engagement de Noskowski écrivain n'était pas purement désintéressé, car il avait un intérêt personnel à imposer en Pologne le concept de musique qu'il avait adopté à Berlin pour y faire carrière. Lorsqu'il rédigea sa première série d'articles, intitulée " Panneaux indicateurs » (Noskowski 1879), il travaillait encore à l'étranger, en tant que chef de chœur à Constance, et cherchait un poste de premier plan en Pologne. Le sous-titre «Esquisses et causeries dans le domaine de la musique » devait masquer la tendance assez rigoureuse de ces articles. Noskowski y brossait une image très négative de la vie musicale à Varsovie qui se trouvait, selon lui, enfermée dans un cercle vicieux nourri par l'incompétence des musiciens et critiques, et par l'ignorance du public. Il présentait ensuite son idéal d'une musique qui serait un besoin humain essentiel, un élément indispensable de la société, de l'éducation scolaire et générale. Finalement, il promouvait une réforme complète de la musique polonaise selon le modèle allemand.

Si cette orientation pouvait repousser les lecteurs, eu égard à l'occupation d'une grande partie du territoire polonais par les pouvoirs austro-allemands, elle était néanmoins assez courante en Pologne à l'époque. Depuis l'échec de l'Insurrection de 1863-1864, la plupart des politiciens et des intellectuels non émigrés avaient adopté le programme du Positivisme varsovien selon lequel il fallait d'abord élever considérablement le niveau économique, culturel et spirituel de la nation avant d'aborder une nouvelle insurrection. Dans ce cadre, il paraissait légitime, voire nécessaire, de s'approprier des savoirs et des compétences à l'étranger, y compris chez les pouvoirs occupants, afin de les surpasser.

Quant au contexte médiatique des textes de Noskowski, quelques-uns parurent dans Echo muzyczne, la seule revue musicale publiée en Pologne à l'époque : fondée en 1877, elle avait rapidement élargi son titre en Echo muzyczne, teatralne $i$ artystyczne ("Écho musical, théâtral et artistique ») afin d'intégrer des articles sur les autres arts ainsi que des romans-feuilletons, et d'attirer un public plus large. Selon Magdalena Dziadek, ce périodique varsovien tirait à 1100 exemplaires en 1896 (Dziadek 2002b, p. 193). Noskowski publia d'autres articles dans des périodiques plus généraux comme Tygodnik ilustrowany ${ }^{1}$, Wędrowiec ${ }^{2}$ ou Świat $^{3}$. Il se servit de ces médias pour cibler un public plus large et plus influent que ne l'était le monde purement musical.

\footnotetext{
1 «Hebdomadaire illustré », fondé en 1859 ; tirage de 7000 exemplaires en 1887, de 19000 en 1904 (toutes les dates selon Dziadek 2002b).

2 "Le Voyageur ", 1863-1906, d'abord un hebdomadaire ethno-géographique ; tirage de 9500 exemplaires en 1896.

3 «Le Monde», 1888-1895, revue bimensuelle scientifique et littéraire avec un tirage de 500 exemplaires.
} 
Dans ses articles, Noskowski n'évoquait que rarement des aspects politiques de façon directe - et ce, à cause de la censure. Notons que même les titres programmatiques des quatre mouvements ${ }^{4}$ de sa Symphonie $n^{\circ}$ 2, en do mineur (1876-1879), qui anticipe la libération nationale de la Pologne à l'aide de la citation et de la transformation caractéristique d'un chant patriotique polonais, n'étaient pas publiés à Varsovie. Ce n'est que lors d'un concert à Cracovie, en secteur autrichien où régnait une politique culturelle plus libérale depuis 1867, que Noskowski osa imprimer sur le programme le titre des quatre mouvements en 1883.

Néanmoins, un concert polonais à Paris en 1887 incita Noskowski à se prononcer publiquement au sujet de l'élément national en musique. Il s'agissait d'un concert de musique de chambre du violoniste Wladyslaw Górski présentant des œuvres contemporaines (Noskowski, Żeleński et Paderewski). Par ce moyen, on voulait démontrer aux Parisiens que la Pologne disposait elle aussi d'une musique instrumentale d'un niveau équivalent à celle des autres pays. Mais dans son compte rendu pour Echo muzyczne, l'écrivaine Marya Szeliga estima qu'on aurait dû choisir plutôt des œuvres plus courtes et vocales pour mieux faire ressortir le caractère national polonais (Szeliga 1887). Noskowski répondit dans un article intitulé "La marque nationale en musique " (Noskowski 1888a). Il admit que l'on pouvait certes se servir d'éléments folkloriques précis tels que des rythmes de Mazurka ou bien la quarte augmentée pour évoquer le caractère polonais. Mais au fond, la musique nationale serait surtout le produit du génie individuel de grands artistes comme Chopin. Certains éléments créés par ces derniers pourraient être rétrospectivement interprétés par leur peuple comme son style national. Cette position était rare dans le discours national de l'époque qui tendait de plus en plus, dans tous les pays, à une mystification du génie collectif des prétendues races.

\section{PADEREWSKI : LA MUSIQUE AU SERVICE DE LA PATRIE}

En Pologne, la conception essentialiste d'une musique nationale fut défendue notamment par Ignacy Jan Paderewski (1860-1941), un représentant emblématique non seulement de la musique, mais aussi de la politique polonaise, car il fut véritablement un homme d'État pendant quelques années, le premier ministre de la Pologne ressuscitée en 1918 - le Traité de Versailles porte notamment sa signature. De Paderewski, nous disposons uniquement d'une édition de ses écrits politiques (Paderewski 1992). Néanmoins, ce sont bel et bien des écrits d'un compositeur, car avant de se lancer dans une carrière mondiale de pianiste, Paderewski avait reçu une formation sérieuse en composition, à Varsovie et ensuite à Berlin, auprès de Friedrich Kiel et Heinrich Urban. Il a composé une trentaine d'œuvres dont un opéra, une symphonie et deux œuvres concertantes avec piano. Admettons que Paderewski représente un cas particulier, puisqu'il écrivit dans diverses langues étrangères et passa la majeure partie de sa vie hors de la Pologne. Il ne prit d'ailleurs sa plume d'écrivain qu'occasionnellement.

4 «1. Le peuple dans la servitude ; 2. Espoir et appel aux armes ; 3. Élégie aux héros tombés ; 4. Per aspera ad astra! ». Voir Keym 2009, p. 24. 
$\mathrm{Au}$ cours de ses études à Berlin, Paderewski publia deux comptes rendus dans la revue Echo muzyczne sur la vie musicale dans la capitale prusso-allemande (Paderewski 1884). Il remarqua que les Allemands étaient opposés au concept de musique nationale et qu'ils semblaient exiger de la part des compositeurs étrangers non seulement qu'ils adoptent les moyens techniques du style allemand, mais encore qu'ils " germanisent » le contenu de leurs œuvres.

La prise de position la plus fameuse de Paderewski sur les rapports entre musique et nation date d'un tournant décisif de sa vie lorsque le célèbre pianiste commença sa carrière politique. En 1910, il finança et inaugura un monument cracovien à la mémoire du cinq-centenaire de la victoire du roi polonais sur l'ordre teutonique à Grunwald ; à cette occasion, il tint son premier discours politique public (Paderewski 1992, p. 58). En fait, les deux carrières de Paderewski (pianiste et politicien) se fondaient toutes deux sur son aura, sa présence personnelle et sa rhétorique. Quelques mois plus tard, il prononça un autre discours lors de la première Assemblée nationale des musiciens polonais, tenue à Lwów (Léopol ou Lviv, aujourd'hui en Ukraine) pour commémorer le centenaire de la naissance de Chopin (soulignons que ces deux manifestations patriotiques eurent lieu dans le secteur autrichien du pays). Dans son discours tenu à Lwów, Paderewski évoqua les rapports entre Chopin et sa patrie (Paderewski 1911a, b, c). Il affirma que même un Polonais non musicien qui ne comprendrait pas les œuvres des grands compositeurs étrangers, serait nécessairement ému par la musique de Chopin, car il y entendrait « la grande voix de sa race » (Paderewski 1911b, p. 12). Selon Paderewski, la musique était liée aux "sentiments et états d'âme des peuples » : la musique polonaise serait imprégnée d'une " arythmie nationale " typiquement polonaise, une tendance à changer rapidement d'un extrême émotionnel à l'autre (ibid., p. 8). Ce tempérament se traduirait notamment par le tempo rubato : « la Pologne entière vit, sent, agit in tempo rubato " (ibid., p. 9). Il évoqua aussi le problème de la censure : "Tout nous fut interdit. La langue de nos mères, la foi de nos aïeux, le culte de notre passé, nos costumes, nos usages, nos poètes [...]. On ne nous laissa que Chopin. Et en Chopin cependant, tout ce qu'on nous défendait se retrouve " (ibid., p. 13-14). À la suite de Schumann, Paderewski appela Chopin « le contrebandier qui dans les feuillets de sa musique fit s'envoler par-dessus les frontières le polonisme prohibé ». Et il alla jusqu'à proclamer que le compositeur polonais " fut le prêtre qui nous porta, dans la dispersion, le sacrement de la Patrie » (ibid., p. 14).

Si cette profession de foi musiconationaliste parait assez exagérée aujourd'hui, il s'agit pourtant d'un document important de la politisation de la musique qui témoigne du prestige symbolique que de simples pièces pour piano pouvaient acquérir dans un contexte politique particulier. Bien entendu, ce texte ne put être publié dans le secteur russe de la Pologne. Mais il circula néanmoins dans des espaces médiatiques importants. Paru dans les actes de l'assemblée de Lwów, il fut également publié à part en plusieurs langues. Une traduction française de Paul Cazin fut imprimée par l'Agence polonaise de presse (ibid.). Dans une autre version publiée dans la Revue musicale de Jules Combarieu, quelques remarques critiques sur la Russie et la France furent supprimées (Paderewski 1911c). À l'heure de l'alliance franco-russe, le patriotisme polonais était aussi mal vu en France qu'ill'avait toujours été en Allemagne. Dans la partition de la Symphonie en si mineur de Paderewski, imprimée en 1911 à Paris 
par Heugel et qui anticipe, comme celle de Noskowski, la libération nationale du pays par le travail thématique et caractéristique sur un motif tiré d'un chant, patriotique, on ne trouve aucune trace verbale de son programme politique.

Grâce à sa gloire de pianiste et à sa fortune internationales, Paderewski disposa de moyens beaucoup plus vastes que les autres compositeurs polonais pour diffuser ses textes et ses idées. Ses mémoires furent publiées en 1939 aux États-Unis en anglais (voir Paderewski et Lawton 1939). Il y confirma son image d'un artiste qui aurait toujours été un grand patriote ("I was born a patriot», ibid., p. 16). Mais même dans ses écrits, la présence de l'aspect politique dépendait du contexte. On peut le voir par exemple dans son chapitre sur le tempo rubato tiré de l'ouvrage collectif Success in Music and How it is Won, publié en 1909 à New York par Henry T. Finck, un critique musical américain et fervent adorateur de Paderewski. Dans ce texte, le tempo rubato n'est pas du tout présenté comme un phénomène exclusivement polonais. Tout au contraire, Paderewski le recommande pour « toute musique nationale » (de la danse hongroise à la valse viennoise), et pour l'interprétation des œuvres de la plupart des compositeurs, notamment des romantiques (Paderewski 1909, p. 454-461).

\section{SZYMANOWSKI : LE NATIONALISME AU SERVICE D'UN NOUVEAU STYLE}

L'impact de contextes spécifiques sur la tendance politique des écrits de compositeurs et sur le choix de leurs espaces médiatiques se voit aussi à travers les écrits de Karol Szymanowski (1882-1937). Cet artiste faisait partie de la génération de la "Młoda Polska », la " Jeune Pologne » qui prônait au début du Xx ${ }^{\mathrm{e}}$ siècle une esthétique moderniste et cosmopolite de "l'art pour l'art » et ne s'intéressait guère aux anciens discours patriotiques ${ }^{5}$ - une position fustigée par Paderewski dans son discours à Lwów. Or, il est intéressant de noter comment Szymanowski arrive à intégrer quelques éléments de la rhétorique nationaliste dans ses écrits des années 1920, et ce dans un but bien différent de celui de Paderewski.

Les écrits de Szymanowski se divisent en textes poétiques et articles sur la musique (Szymanowski 1984 et 1989) ${ }^{6}$. Parmi les premiers, notons surtout son roman à clé homoérotique Efebos qui exprime sa vision du monde et de 1'art. Malheureusement, le manuscrit de ce roman fut détruit pendant la Seconde Guerre mondiale et il n'en reste qu'un chapitre central, le symposion (Szymanowski 1984, p. 141-168).

La carrière de Szymanowski journaliste ne commença que dans les années 1920, après son retour en Pologne dont il avait été séparé pendant la Première Guerre mondiale, car sa ferme domaniale était située tout à 1'Est, en Ukraine. Pendant cette période d'isolement, Szymanowski changea profondément son style qui avait été fortement enraciné dans la tradition allemande, grâce à l'enseignement qu'il avait reçu de son oncle Gustav Neuhaus et de Noskowski. La découverte des ballets russes de Stravinski incita Szymanowski à développer, lui aussi, un style nouveau, fondé sur

5 Pour l'esthétique de Szymanowski, voir Moere 2008.

6 La plupart des écrits musicaux viennent d'être traduits en français dans Szymanowski 2018. 
des ostinati et des mélopées orientales. Après la fuite de sa famille devant le nouveau régime bolchévique en Ukraine et son arrivée à Varsovie, le but de Szymanowski était de faire connaître ses œuvres récentes au public polonais et de trouver sa place dans le nouvel État indépendant polonais. Et comme Noskowski, il avait besoin des émoluments que lui procurait l'écriture de ses articles, ayant laissé tous ses biens en Ukraine.

C'est dans ce contexte qu'il faut interpréter les articles de Szymanowski. Le premier fut publié à l'été 1920 dans une revue mensuelle littéraire et artistique et faisait état de la situation actuelle et de l'histoire de la musique polonaise ${ }^{7}$. Szymanowski dressait un panorama très sévère : selon lui, il n'y existait pas de tradition nationale musicale, car les compositeurs polonais n'auraient pas compris la leçon de Chopin - à la différence de leurs confrères russes. Ils auraient préféré une adaptation académique d'éléments folkloriques, en écrivant des fugues sur des chants populaires. Si ce texte atteste l'attitude antiacadémique de Szymanowski (qui n'avait pas terminé la classe de Noskowski au Conservatoire de Varsovie), il implique aussi une prise de distance par rapport aux modèles germaniques. Néanmoins, le compositeur fustige également la mauvaise habitude des critiques musicaux polonais de se servir de l'argument national pour toucher leurs adversaires, et notamment pour attaquer tout nouveau courant.

Szymanowski adopta d'ailleurs lui-même cette stratégie par la suite, mais dans le sens opposé, c'est-à-dire pour défendre son nouveau style. En 1922, la création de son Concerto pour violon $n^{\circ} 1$ déclencha une querelle dans la presse polonaise. Des critiques conservateurs lui reprochèrent un manque de caractère symphonique et de profondeur spirituelle. Ce jugement négatif fondé sur des critères tirés de la tradition allemande incita Szymanowski à passer à l'attaque : dans un entretien avec le quotidien Kurier Polski, il déclara que la longue ère de l'hégémonie allemande en musique était définitivement finie, car on remarquait dans de nombreux pays toute une série de "rébellions ", de Chopin et Moussorgski jusqu'à Debussy, Ravel, Prokofiev et le Groupe des Six. L' " exotisme barbare » de Stravinski et la " clarté latine » de la nouvelle école française, entre autres, avaient selon lui mis fin à tous les clichés de l'esthétique officielle du XIX siècle et à leur prolongement dans le style surchargé et confus d'un Strauss et d'un Reger ${ }^{8}$.

Szymanowski se fit donc l'avocat du changement stylistique qui se produisit un peu partout au cours années 1920, changement du postromantisme vers un néoclassicisme se fondant souvent sur des éléments folkloriques. Dans ce but, il recourut à des arguments politiques : en identifiant le style dépassé du XIX siècle à la musique germanique, il établit une analogie entre la prétendue hégémonie de ce style et l'occupation politique et militaire de la Pologne par la Prusse et l'Autriche. Dans cette

7 «Uwagi w sprawie współczesnej opinii muzycznej w Polsce » [Remarques au sujet de l'opinion musicale contemporaine en Pologne], Nowy Przeglad Literatury $i$ Sztuki, juin 1920, repris dans Szymanowski 1984, p. 33-47.

8 «Karol Szymanowski o muzyce współczesnej » [K. S. sur la musique contemporaine], Kurier Polski, 22 novembre 1922, repris dans ibid., p. 58-62. 
optique, la lutte pour l'émancipation de ce style ancien devint un élément nécessaire à la libération culturelle et spirituelle de la nation polonaise. Les compositeurs polonais devaient, eux aussi, contribuer à cette libération. Et ils devaient avoir des alliés éminents à l'Ouest ainsi qu'à l'Est. On trouve une argumentation politisée similaire chez des compositeurs néoclassiques de nationalités diverses, non seulement en France (Groupe des Six), mais encore chez Alfredo Casella, Bohuslav Martinů ou bien Aaron Copland. À leur différence, Szymanowski était beaucoup plus enraciné dans la tradition allemande, ce qu'il avoua lui-même en parlant de sa lutte personnelle avec « le cauchemar de la tradition académique » (Szymanowski 1984, p. 69).

Sur la base de cette compétence et de cette expérience personnelles, Szymanowski se sentait la vocation de propager son nouveau style personnel comme la voie unique par laquelle la musique polonaise pourrait arriver à une originalité, une qualité et une modernité nouvelles (il reprit en quelque sorte l'idée de Noskowski sur le rapport entre génie individuel et style national). Il réclama une sorte de leadership d'opinion dans le discours musical de la nouvelle Pologne. Cette ambition se perçoit à travers le choix des espaces médiatiques dans lesquels il se prononça : son entretien ainsi que les deux articles suivants de 1922-1923 furent publiés dans de grands quotidiens destinés à un lectorat de masse ${ }^{9}$. Et le fait que ces journaux lui offrirent de la place pour ces textes assez longs et complexes témoigne de l'importance que l'on attribuait à sa voix dans l'espace public de l'époque. En cela, il profitait du prestige national dont les artistes bénéficiaient dans la société polonaise depuis la période de l'occupation.

Szymanowski s'efforça de consolider sa position par d'autres articles : sur les différents courants de la musique contemporaine, sur ses compositeurs préférés (Chopin, Stravinski, Ravel), sur sa rencontre avec le Groupe des Six, sur ses rapports avec le romantisme et sur la musique folklorique des Górales, les habitants montagnards des Tatras. Ces articles parurent dans des quotidiens, des revues d'avantgarde (Skamander) et des journaux littéraires (Wiadomośći literackie), dans la revue musicologique Muzyka ou encore dans le magazine féminin Pani.

Cette campagne publicitaire trouva surtout un écho auprès des jeunes compositeurs en les encourageant à chercher de nouveaux chemins. Mais ceux-ci allaient bientôt dépasser leur modèle et commençaient à reprocher à Szymanowski de rester à cheval entre la musique des $\mathrm{XIX}^{\mathrm{e}}$ et $\mathrm{XX}^{\mathrm{e}}$ siècles. Aujourd'hui, on comprend mieux que c'est précisément dans ce positionnement que réside l'individualité de Szymanowski. Mais à l'époque, l'idéologie de la pureté, en musique ainsi qu'en politique, primait et Szymanowski lui-même avait coutume dans ses textes de relier pureté de style et pureté de race. Là encore, tout dépend du contexte, car il existe aussi un texte de sa plume dans lequel il reprend l'idéalisme humaniste et international propagé déjà par Noskowski 50 ans plus tôt. Ce long article sur « Le rôle éducatif de la culture musicale dans la société » fut publié dans la revue générale Pamiętnik warszawski par Szymanowski après sa nomination inattendue en 1930 au poste de directeur de la nouvelle Académie de musique à Varsovie (ibid., p. 264-292).

9 "My splendid isolation », Kurier Polski, 26 novembre 1922, et « Opuszczę skalny mój szaniec » [Je quitterai mon bastion de pierre...], Rzeczpospolita, 8 janvier 1923, repris dans Szymanowski 1984, p. 67-88. 
Si ce propos officiel parait presque incompatible avec la polémique nationaliste des textes précédents, Szymanowski arriva à un équilibre fragile entre ces deux pôles opposés dans son discours sur "L'avenir de la culture ", prononcé en français à Madrid en mai 1933 au congrès de l'Institut international de coopération intellectuelle de la Société des Nations, où il fut le seul représentant de la musique. Dans ce texte, Szymanowski insista sur le "développement prodigieux des écoles nationales " dans la musique contemporaine et critiqua de nouveau " la légende de l'universalité de la musique allemande » dont le "sens démesuré des proportions, son pathos romantique et transcendant " auraient suggéré aux autres nations " une vision de valeur unique ", en dehors de laquelle " toute autre conception de la musique semblait puérile et vaine »(Szymanowski 1984, p. 340-341). En fait, ce concept d'un style musical universel avait été très répandu dans le discours musical allemand (de Franz Brendel à Hugo Riemann). Des auteurs polonais tels que Paderewski, Szymanowski et la musicologue Zofia Lissa furent parmi les premiers à dénoncer le nationalisme caché de ce concept, qui présuppose l'idée de l'hégémonie d'une nation hébergeant l' " esprit du monde " (le Weltgeist selon Hegel) pendant une époque donnée. Mais Szymanowski souligna aussi que le retour nécessaire aux « sources mêmes de la sensibilité de telle nation » dans les années 1920 aurait finalement produit « un besoin de connaissances et de rapprochements mutuels ", et il demanda d'" abolir ces douanes spirituelles qui [...] s'opposent à une compréhension mutuelle des peuples » (ibid., p. 341-342). Le texte culmine dans l'hypothèse qu'à la base de toute activité artistique repose « un sentiment profondément et universellement humain » et dans l'espoir que " la sphère pure et désintéressée de l'art » pourrait apporter « une résolution idéale de l'antinomie apparente des cultures nationale et humaine » (ibid., p. 342).

Même si Szymanowski n'arrive pas à y résoudre complètement les contradictions inhérentes à ses textes de l'entre-deux-guerres, cet article est un témoignage important d'une nouvelle étape dans la réflexion du compositeur sur les rapports entre musique et politique - et ce, trois mois après la prise de pouvoir par les nazis et trois ans avant qu'éclate la guerre civile espagnole.

Du point de vue de la musique pure et de «l'art pour l'art », l'habitude consistant à mêler idées politiques et esthétiques, typique du discours artistique polonais des XIX et $\mathrm{XX}^{\mathrm{e}}$ siècles, peut être perçue comme une alliance dangereuse, un pacte avec le diable ou bien une forme de prostitution de l'art. Mais l'idéologie de la "musique pure » comporte également des aspects politiques sous-jacents. En tout cas, la situation particulière des compositeurs polonais a incité ces derniers à formuler des hypothèses sur les rapports entre musique et politique, musique et nation, musique et société, hypothèses qui sont pertinentes aussi dans d'autres contextes géographiques ; ce positionnement les a amenés à investir des espaces médiatiques importants, tant à l'échelle nationale (y compris dans des supports lus par leurs compatriotes non musiciens) qu'à l'échelle internationale. Les textes de Noskowski, Paderewski et Szymanowski méritent ainsi plus d'attention en dehors des frontières de leur pays que celle qu'on leur a consacrée jusqu'ici. 


\section{BIBLIOGRAPHIE}

Demska-Trębacz, Mieczysława (dir.) (1991), Muzyka i naród. Wybór tekstów [Musique et nation. Textes choisis], Warszawa, Akademia muzyczna F. Chopina.

Dziadek, Magdalena (2002a et b), Polska krytyka muzyczna w latach 1890-1914 [La critique musicale polonaise dans les années 1890-1914], "Vol. 1. Koncepcje i zagadnienia " [Conceptions et problèmes], Katowice, Wydawnictwo Uniwersytetu Śląskiego ; "Vol. 2. Czasopisma i autorzy » [Périodiques et auteurs], Cieszyn, Uniwersytet Śląski.

Jarociński, Stefan (dir.) (1955), Antologia polskiej krytyki muzycznej XIX $i$ XX wieku [Anthologie de la critique musicale polonaise des $\mathrm{XIX}^{\mathrm{e}}$ et $\mathrm{XX}^{\mathrm{e}}$ siècles], Kraków, PWM.

Keym, Stefan (2009), "The Tradition of 'per aspera ad astra' in Polish Symphonic Music from Zygmunt Noskowski to Karol Szymanowski », Muzyka, vol. 54, n 3-4, p. 21-44.

Keym, Stefan(2010), Symphonie-Kulturtransfer. Untersuchungenzum Studienaufenthaltpolnischer Komponisten in Deutschland und zu ihrer Auseinandersetzung mit der symphonischen Tradition 1867-1918 [Symphonie et transferts culturels. Recherches sur les études de compositeurs polonais en Allemagne et sur leur appropriation de la tradition symphonique], Hildesheim, Georg Olms.

Lutosławski, Witold (2011), O muzyce. Pisma i wypowiedzi [Sur la musique. Écrits et entretiens], sous la direction de Zbigniew Skowron, Gdańsk, Slowo/obraz terytoria [éd. anglaise 2007].

Moere, Didier van (2008), Karol Szymanowski, Paris, Fayard.

Noskowski, Zygmunt (1879), «Drogoskazy. Szkice i gawędy z dziedziny muzyki» [Poteaux indicateurs. Esquisses et causeries du domaine de la musique], Echo muzyczne, vol. 3, n 10 , p. $2-3$; no 11, p. 2-3 ; $\mathrm{n}^{\circ} 12$, p. $2-4 ; \mathrm{n}^{\circ} 13$, p. $3-4 ; \mathrm{n}^{\circ} 15$, p. 3-4.

Noskowski, Zygmunt (1888a), «Piętno narodowe w sztuce muzycznej » [L'empreinte nationale dans l'art de la musique], Świat, vol. 1, p. 15-18 et 82-85.

Noskowski, Zygmunt (1888b), "Ideał opery » [L'opéra idéal], Echo muzyczne, teatralne i artystyczne, vol. 5, nº 237-240, p. 169-171, 186-187, 194-196 et 207-208.

Noskowski, Zygmunt (1892), « Od Bacha do Chopina » [De Bach à Chopin], Świat, vol. 5, p. 254-260, 279-282, 308-310 et 323-325.

Noskowski, Zygmunt (1907), Kontrapunkt, kanony, warjacje i fuga. Wyktad praktyczny [Manuel pratique], Varsovie, Gebethner \& Wolff.

Paderewski, Ignacy Jan (1884), Deux correspondances sans titre de Berlin, Echo Muzyczne i Teatralne, vol. $1, \mathrm{n}^{\circ} 18$ et $26, \mathrm{p} .192-193$ et $272-273$.

Paderewski, Ignacy Jan (1909), "Tempo rubato », dans Henry T. Finck, Success in Music and How it is Won, New York, Charles Scribner's Sons, p. 454-461, https://dicteco.huma-num.fr/fr/ workchapter/40692, consulté le 7 août 2019.

Paderewski, Ignacy Jan (1911a), O Szopenie. Mowa wygtoszona na obchodzie szopenowskim w Filharmonii dnia 23 października 1910, Lwów, E. Wende.

Paderewski, Ignacy Jan (1911b), À la mémoire de Frédéric Chopin. Discours prononcé le 23 octobre 1910 à Léopol, Paris, Agence polonaise de presse.

Paderewski, Ignacy Jan (1911c), « Frédéric Chopin », Revue musicale, no 11, p. 247-250.

Paderewski, Ignacy Jan (1992), Myśli o Polsce i Polonii [Pensées sur la Pologne et la Polonia], sous la direction de Marian M. Drozdowski et Andrzej Piber, Paris, Ed. Dembinski.

Paderewski, Jan Ignacy, et Mary Lawton (1939), The Paderewski Memoirs, New York, Charles Scribner's Sons ; Londres, Collins, https://dicteco.huma-num.fr/fr/book/40680, consulté le 7 août 2019.

Szeliga, Marya (1887), " Z powodu koncertu Władysława Górskiego » [À propos du concert de Władysław Górski], Echo muzyczne, teatralne i artystyczne, no 176, p. 74-75.

Szymanowski, Karol (1984), Pisma muzyczne, sous la direction de Kornel Michałowski, Kraków, Polskie Wydawnictwo Muzyczne. 
Szymanowski, Karol (1989), Pisma poetyczne, sous la direction de Teresa Chylińska, Kraków, Polskie Wydawnictwo Muzyczne.

Szymanowski, Karol (2018), Écrits sur la musique, trad. par Christophe Jezewski et Charles-Henri du Bord, Lyon, Symétrie.

Tomaszewski, Mieczysław (dir.) (1959), Kompozytorzy polscy o Fryderyku Chopinie [Compositeurs polonais sur Frédéric Chopin], Kraków, Polskie Wydawnictwo Muzyczne [éd. anglaise 2002]. 